\title{
The Role of Drones in Ambient Assisted Living Systems for the Elderly
}

\author{
Radosveta Sokullu (D), Abdullah Balc1 (D), and Eren Demir ${ }^{(\triangle)}{ }_{(\mathbb{D})}$ \\ Department of Electrical and Electronics Engineering, \\ Ege University, Izmir, Turkey \\ radosveta.sokullu@ege.edu.tr, erendemir33@gmail.com
}

\begin{abstract}
Following years of restricted military applications, recently unmanned aerial vehicles (UAV) also known as drones have become the new disruptive force in many industrial and everyday life applications. Very obvious usage areas are transportation and parcel delivery. However, recent research reveals that there are a number of ways that drones can be employed to help elderly people sustain a better independent lifestyle.

This paper introduces some of the most recent and interesting applications that drones can find in creating ambient assisted living environments for the elderly. Advantages and disadvantages, possible healthcare models and challenges are discussed. Even though these are some very interesting and original applications there are a lot of challenges involved in accepting drones as "flying assistants" to extend the independent living environment of the elderly.
\end{abstract}

Keywords: Ambient assisted living systems - Unmanned aerial vehicles Elderly $\cdot$ Drones $\cdot$ Daily activities monitoring

\section{Introduction}

Extending lifespan and increased aging population in many countries around the world present a considerable challenge for healthcare services and systems. The two main options - personal care at home or in care in a nursing facility - are very human labor demanding and place a huge financial burden both for the elderly and their relatives, and the healthcare system. Recent advances in networking, computing and sensor technologies have helped address these issues in an efficient and cost-effective way while extending the independent life of the elderly. In the past two decades numerous research projects have focused on various aspects of ambient assisted living systems. (AALS) - from sensor networks to context aware data services - trying to provide endto-end solutions. Today there are many IoT based applications which ensure continuous and often real-time monitoring of the occupant and his surrounding environment, providing assessment and triggering assistance when necessary. AALS is a growing area of research where new enabling technologies help add new features and solve new challenges. Unmanned aerial vehicles, commonly known as drones, are one such technology. For years they have been indispensable for many military applications, but recently they have claimed their place in our everyday lives as well. From weather probing and gaming, to photography and transportation, drones have quickly become 
the new disruptive force in our society. Could they also add something new to AALS? How will the conservative elderly accept these flying mini robots? There are numerous questions that can come to one's mind.

In this chapter we first introduce the drones - their structure, features, general capabilities and limitations. Then we discuss the main characteristics of AALS, technologies and protocols. The third section presents an overview of existing drone based applications for ambient assisted living followed by a critical analysis and evaluation of drone related technologies and their relationship with ambient assisted living for the elderly.

\section{Unmanned Aerial Vehicles - Structure and Main Features}

\subsection{Structure and Functionality of Unmanned Aerial Vehicles}

Unmanned Aerial Vehicles (UAV), simply known as "drones" can be defined as small size vehicles capable of flying by utilizing air currents and driving forces, capable of autonomous flight, capable of carrying loads and can be controlled remotely. In general, an UAV flight system consists of five basic components as seen in Fig. 1. The first basic component is the set of sensors such as a barometer, a pitot tube, a current/voltage sensor, an IMU (inertia measurement unit), distance, temperature and magnetometer sensors. The barometer sensor provides altitude information by sensing pressure, while the pilot tube is generally incorporated in fixed wing aircrafts and is useful in measuring air velocity. This information is further used for flying at stall speed. Stall speed is the minimum speed the aircraft must fly without losing its altitude. The IMU sensor provides three or six axis gyro stabilization information to the flight controller and it is one of the most important and essential parts of an UAV flight control system. Distance sensor measures the distance between UAV and surrounding obstacles, thus providing information to avoid collision with other objects. Localization and navigation is very important for an UAV. Therefore, in most cases UAVs use magneto sensor and GPS satellites for navigation and localization. However, in closed places like inside buildings and sometimes in very rugged terrain, it is impossible or very difficult for an UAV to navigate because of non-existing or weak GPS signal. Thus, in some application scenarios more robust location system design might be required. One of the most important parameters affecting the wing lifting power of airplanes is pressure and temperature. Therefore, the temperature sensor provides the flight controller with the necessary information for the lift force account.

The second main component is the autopilot which interprets the information from all the other components, keeps the aircraft in balance and allows it to fly. For the motors to reach the desired speed it sends commands to the electronic speed controller (ESC). In addition, the autopilot receives commands from the remote controller and according to the information received it controls the servos and other motors. The third component is the communication unit which is also divided into three sub-components; remote controller, telemetric module and video transmitter. The Remote Controller (RC) unit is capable of transmitting control commands to the UAV. The communication link of the RC unit uses $433 \mathrm{MHz}, 2.4 \mathrm{GHz}$ or $5.8 \mathrm{GHz}$. Each frequency can be 


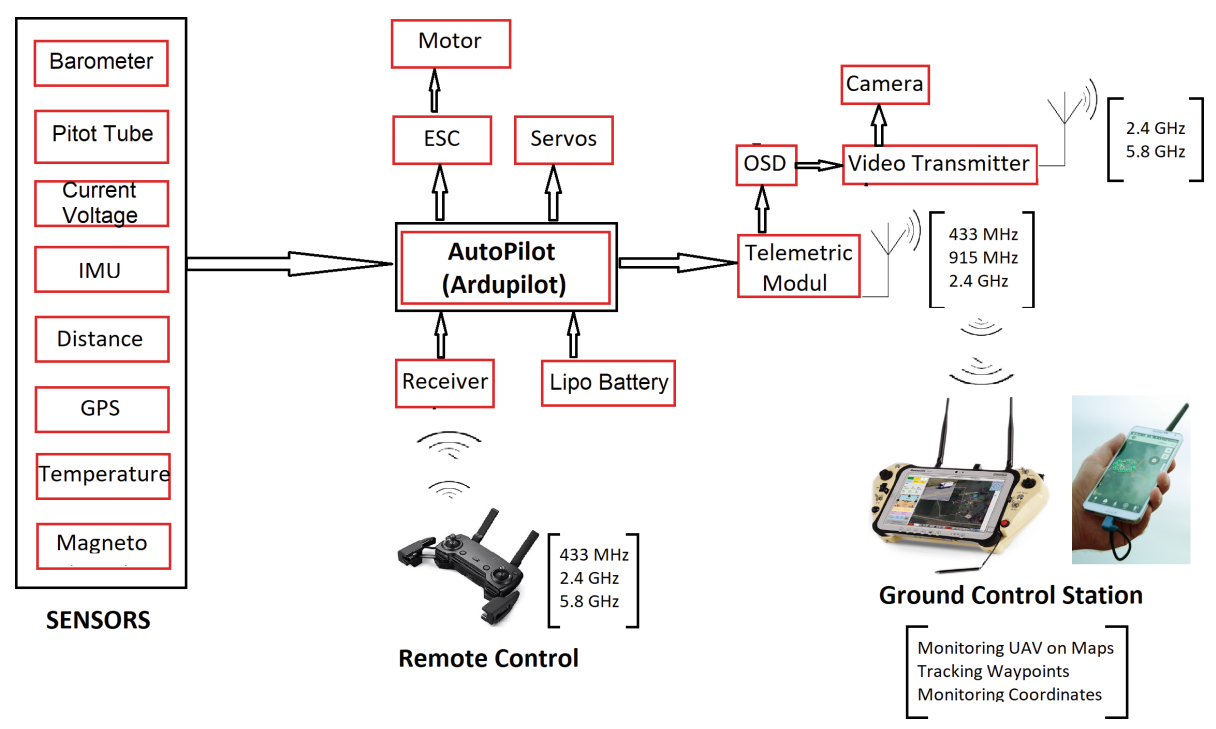

Fig. 1. Basic components of an UAV

used to meet different application requirements. UAVs can fly autonomously or with pilot (human) remote control navigation. In order to ensure safety, UAVs are obliged to be continuously in the RC coverage area, a rule regulated by law. The telemetric module sends the information from the sensors to the ground control station for evaluation. Also, if desired, sensor information can be superimposed on the camera image via the OSD (on screen display) module and sent to the GCS (ground control station) via the video transmitter. The fourth component is the GCS which displays real time data for the UAVs location and performance. It is also used to montitor live stream data generated by the UAV camera. The fifth and last main component is the battery. Lithium polymer (LiPo) is used almost entirely in batteries for UAVs, offering high capacity with low weight and high discharge rates. However, it has some disadvantages such as higher cost and ongoing safety issues.

\subsection{Classification of UAVs}

UAVs are categorized either based on their flight capabilities or on their structure. The categorization is given in Fig. 2. As far as flight capabilities are considered there are three main UAVs which are HALE, MALE and VTOL. HALE (high altitude long endurance) can fly over $9000 \mathrm{~m}$ and has long flight endurance whereas MALE (medium altitude long endurance) can fly up to $9000 \mathrm{~m}$. VTOL (vertical take-off and landing) has the ability of vertical take-off and landing. In addition, after rising up to a specific altitude above sea level, VTOL can move to horizontal flight by the action of the propeller. Considering the configuration, the UAVs can be divided in three categories "fixed-wing", "multi-rotor" and "flap wing". Fixed wing UAVs have greater range and endurance when compared to multi-rotor ones, however multi-rotor UAV 
have the ability to hover and are easier to use. On the flip side, multi-rotor UAVs have greater drain on the battery. Flap wing UAVs are known to experience difficulties in the autopilot design because the wings have to support the control of movement in pitch, yaw and roll direction.

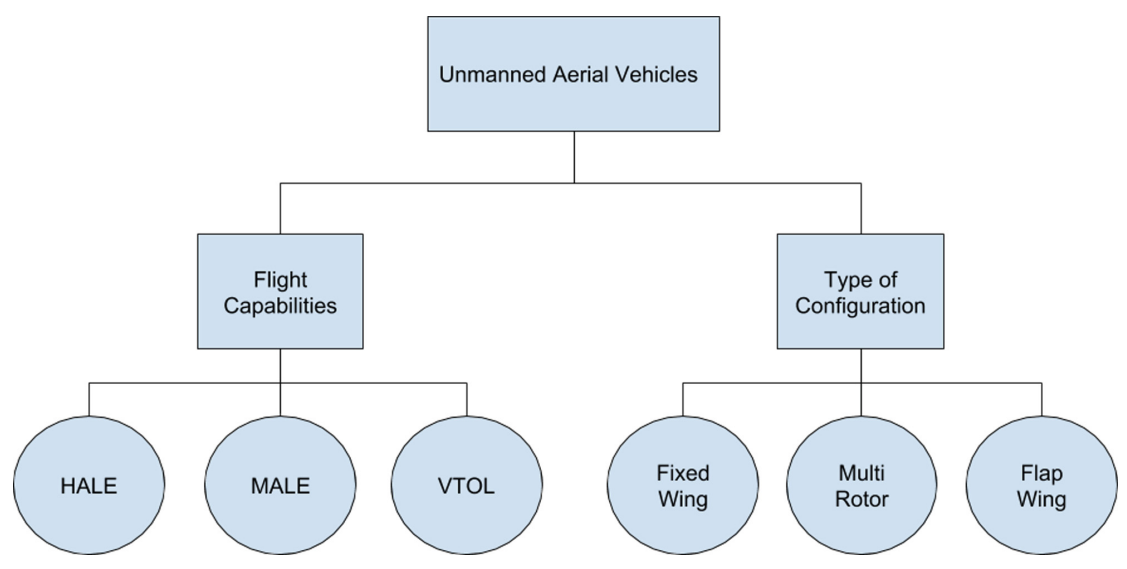

Fig. 2. Unmanned aerial vehicle categorization

In the literature there are a lot of examples related to the applications of UAV and general usage fields can be summarized as: civil transport and transportation, scientific monitoring, geological and ocean surveys, atmospheric research and weather forecasting, surveillance and monitoring (hurricane, volcanic, oil spills, earthquakes, landslides, coast protection, pipelines, water resources, water pollution), international border patrol and drug traffic control, emergency situations (search and rescue, firefighting, disaster operation management, post-disaster site scanning) control of high voltage lines, aerial photogrammetry, detection and analysis of archaeological sites, Earth mapping and 3D city/terrain modeling. Adding to this long list of applications we have also found that very recently drones are finding their place in applications that allow the elderly to live longer full, active lives - both physically and mentally.

\section{Ambient Assisted Living - Technological and System Architecture Issues}

\subsection{Technologies for AALS}

Assisted living technologies have become a very popular research area in the recent years because of providing more helpful and life enhancing approaches to the challenges in daily life of the elderly. As a rule, each assisted technology is specialized according to the application it addresses to perform a specific task. For instance, wearable technology such as eyeglasses, [1], or watches aim to improve the quality of 
life for people interested in fitness and physical exercises. In a similar way, ambient assisted living technologies address the challenges of an aging population.

A very in depth review is presented in [2], where the evolution of AAL technologies is divided into three categories; first, second and third generation technologies. First generation technologies are usually concentrated on emergency response mechanisms. Solutions consist of an alarm system which can be wearable detection sensors like pressure sensor, gyroscope, etc. to detect mainly falls and other life threatening emergency situations. Thanks to such systems and the notifications they send, the uneasiness of families and caregivers about the security and health of their elderly is greatly reduced. However, the wearable devices can be difficult to accept and cause high stress level for the patient himself. Because the older generation in general is not at ease with AAL, they may take off the wearable devices or damage them. In such cases, the alarm may not be triggered even if the patient is stuck in a difficult situation. The second-generation technologies differ from first-generation in terms of the functionalities. The automated systems, which involve home sensors, automatic response to emergencies, aim to reduce the negative effects of first-generation technology by giving less responsibility to the elderly. For instance, if there is a water leakage or flood in the house, the sensors may detect the hazards and automatically trigger the alarm. The third-generation technologies are the integration of first and second-generation technologies which not only monitor the home, but also monitor the physical activities of the person and possibly his environment. In general they are integrated systems which consist of the home automated system and wearable devices and provide continuous monitoring of the vital signs and activities of the elderly patient, reporting the situation to families and caregivers. Actuators play an important role in this technology to improve the quality of life. Actuators can be medical devices such as smart pillbox, or controlling mechanism such as cooker control. Such technologies highly increase the independence of the elderly patient.

AALS rely greatly on IoT-based technologies that support the connection between different devices over the Internet to enable monitoring and remote assisting. IoT has emerged as the new paradigm describing the world of connected physical or virtual things, devices, or machines equipped with sensors and actuators.

\subsection{IoT-Based AAL Architecture}

IoT based architectures are generally designed to ensure the connection between the sensing devices and end-user via gateways. Major standardization institutes, such as 3GPP and IEEE, focus on a general architecture based on comprising a three tier IoT structure: sensing layer, network layer, and application layer as shown in Fig. 3 [3]. The lowest layer, comprising different sensor units is responsible for the collection of the data from the environment. All data is transmitted through the network via specific gateways to the application layer/server. However, the evaluation of the data is only done at the application layer where the application server makes a decision and extracts the required information from the collected data. 

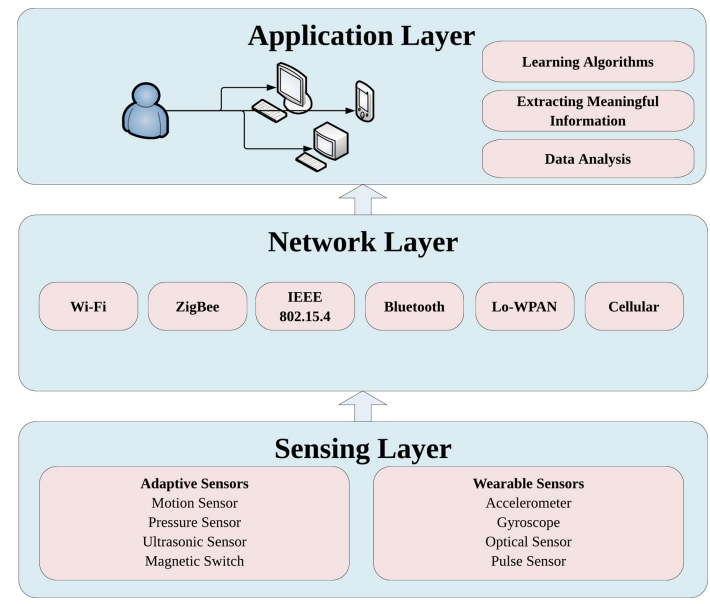

Fig. 3. IoT-based AAL architecture

\section{Sensing Layer}

Sensing equipment is responsible for gathering data about the environment and its inhabitants. This layer plays a big role in the system and constitutes the lower layer of the architecture. Because it is the immediate connection with the environment, lot of attention should be paid to the accuracy of sensors. Further, the sensors should be aggregated to generate more reliable data. In [4], the sensors for the AAL are divided into two main groups: ambient sensors used in smart environments and wearable and mobile sensors. Ambient sensors are generally used for monitoring the environment in order to track the activity of elderly people which are located in a predefined area. Because the ambient sensors are located in the home area, their main advantage is that they do not affect the mobility and comfort of elderly people. Without being exhaustive a list of such sensors is given in Tables 1 and 2.

Wearable and mobile sensors monitor the vital signs of elderly people which are generally incorporated in another electronic equipment such as mobile phones, smart watches, etc. or attached on human body. This type of sensors is used in Body Area Networks (BAN). While most of the smart phones include an accelerometer, gyroscope sensors, there are also some specific models which are even equipped with pulse sensors to measure the hearth rate. Instead of sticking or attaching the sensors to the human body, researchers concentrate on optical sensors to measure the blood pressure [5], heart rate [6], etc. Such an approach will provide a more comfortable sensor platform than most of today's wearable sensors. 
Table 1. Ambient sensors

\begin{tabular}{l|l}
\hline Sensor & Measurement \\
\hline Infrared motion sensor & Motion \\
\hline Radio frequency identification & Object information \\
\hline Pressure & Pressure on chair, bed, tiles \\
\hline Magnetic switches & Door opening/closing \\
\hline Ultrasonic & Motion/distance \\
\hline Camera & Activity \\
\hline Microphone & Activity \\
\hline
\end{tabular}

Table 2. Wearable and mobile sensors

\begin{tabular}{l|l}
\hline Sensor & Measurement \\
\hline Accelerometer & Movements \\
\hline Gyroscope & Motion \\
\hline Optical sensors & Blood pressure \\
\hline Electrocardiography & Cardiac activity \\
\hline Electroencephalography & Brain activity \\
\hline Pulse sensor & Heart rate \\
\hline Thermal & Body temperature \\
\hline
\end{tabular}

\section{Network Layer}

The network layer is responsible for the communication functions and provides a connection between the device with attached sensor(s) and the gateway or center. Different wireless communication technologies can be used to enable the connection between machines, such as Wireless Sensor Networks (WSN), Machine-to-Machine Communication (M2M), Device-to-Device Communication (D2D), Drone-Assisted Communication, etc. The standardization institutes focus on wireless communication architectures for new emerging communication technologies. 3GPP and ETSI have already proposed an architecture for M2M communication over LTE Network which is in line with the AAL architecture. Besides the cellular network, Wi-Fi, ZigBee, IEEE 802.15.4, Bluetooth, Lo-WPAN, Sigfox are other wireless communication techniques that can enable the communication between machines and are expected to be integrated with M2M, D2D or Drone-Assisted Communication. In [7], the authors used ZigBee and IEEE 802.15.4 protocols in their smart home assistant which monitors the activity of elderly people suffering from dementia. An intelligent communication architecture for AAL is presented [8]. In order to monitor the house and control the objects (lamp, water, air conditioner) to detect hazards (high blood pressure, falls, etc.) the authors utilize more than one wireless communication technologies - wireless sensor network, wireless ad-hoc network and wireless mesh network. The purpose of wireless communication technologies is efficiently collecting, exchanging, and transmitting the collected environment data. In case when the sensing unit is wearable and mobile in AAL, the communication technologies should be harmless for the elderly people. 


\section{Application Layer}

The application layer is the highest layer in the stack which is responsible for functions related to software based data processes like aggregation, analysis and generating meaningful information from the incoming sensor data. In the smart home applications, it is very important to be able to define and/or predict the activity of the elderly person by using the data coming from more than one sensor. The prediction of the activity is based on sophisticated learning algorithms which represents an emerging research area in smart home applications $[9,10]$.

The application layer is also responsible for the delivery of services to the end users and providing the necessary user interfaces. Existing projects focus on several categories of AAL applications; checking and reminding daily needs, continuous vital sign monitoring, and emergency detection and notification. Regarding the interfaces it is important that they are both functional and simple in order to be accepted by elderly users which in general have less technological skills than the general user.

\section{AAL Applications Using Drones}

As an important area of research, AALS applications have been discussed and evaluated in many comprehensive review papers. A very recent work in this area is [11] where the authors investigate approaches for developing AALS and identify current practices and directions for future research. Ambient assisted living is defined as the provision of sustainable care for the growing number of elderly in their homes or selected living environments, personalized care based on their profile and surrounding context, which will allow them to extend their independent existence. Having this definition in mind, four major aspects of AAL systems can be defined:

1. Health monitoring aspect.

2. Safety related aspect.

3. Daily activities and routines aspect.

4. Social connectedness aspect.

Historically the health monitoring aspect was the first to be considered and today there are elaborate systems and solutions spanning from monitoring of vital signs and chronic diseases to active telemedicine solutions like remote interaction with patients and assess of health records. What can drones contribute in this aspect? Some interesting projects are considered in Subsect. 4.1.

Safety of the elderly, whether living alone or in a nursing facility has always been a prime concern. Reduced mobility together with increased instability is a common reason for fatal falls and traumas. On the other hand, different emergencies like wandering away and getting lost are also related to the personal safety of the elderly. Fall detection has been a long term research topic and a number of possible response actions especially for elderly living alone have been proposed. [12-14] Can drone technology add something new to these systems? Several very innovative solutions are discussed in Subsect. 4.2.

With increased age people tend to become less active and more detached, even following simple routine daily activities can become a burden. Scientists from the 
medical and the engineering community have joined efforts to enhance the living environments in a way to help and motivate the elderly to continue an active daily life. This is much easier in nursing facilities, where more options exist and patients are more easily motivation for physical activities. For people living alone at home even routine physical exercises can become a considerable issue. Can robots or drones become the new era pets for the elderly that will help motivate them for physical activities? Some very intriguing proposals are covered in Subsect. 4.3.

As several major reviews have concluded, many of the existing AAL project and system solution address these first three aspects. However, the last one, social inclusion and contentedness, is most often overlooked. A major reason stems from the fact that the aging population in general is much less accustomed to the new emerging technologies and the acceptance rate at this late stage can be very low. Being usually physically restricted, as most elderly usually are, naturally but unfortunately unnoticeably leads to social isolation, which in its turn further reduces the motivation for physical activities and starts a vicious downward cycle of exclusion. Will drone technology and applications be more easily acceptable for the elderly to help them keep both physical and social inclusiveness? Some proposed solutions are discussed in Subsect. 4.4.

From here on this section is organized as follows: Subsects. 4.1-4.4 discuss the details of the UAV based AAL applications based on the characteristics defined in Subsect. 4.1. Subsect. 4.5 focuses on the challenges and possible evaluation criteria for these types of applications.

\subsection{Drone Aided Health Monitoring Systems}

According to an extensive study from 2004 and its update in 2012 in the USA [15] the cost of chronic diseases is an overwhelming component for overall healthcare expenses. In the US alone, 117 million people had one or more chronic disease in 2012, which included heart disease, diabetes, arthritis and obesity. A detailed study of medical costs for treating chronic diseases can be found in [16]. Table 3 below gives an idea about the annual cost chronic diseases incur.

Table 3. Annual medical cost for different number of chronic conditions

\begin{tabular}{l|c}
\hline Number of chronic diseases & Annual medical cost (in USD) \\
\hline No chronic diseases & 1117 \\
\hline 1 Chronic disease & 2915 \\
\hline 2 Chronic diseases & 4731 \\
\hline 3 Chronic diseases & 6751 \\
\hline 4 Chronic diseases & 9162 \\
\hline 5 Chronic diseases & 15964 \\
\hline
\end{tabular}

Chronic disease patients are required to visit medical facilities on a regular basis for routine checkups and medicine replenish. Different community solutions and pilot 
programs addressing the elderly combining healthcare with homecare delivery have been initiated. However, the price of these services even at a limited scale is overwhelming, especially for remote and rural areas. Lack of transportation is a major reason for failure of regular medication intake. Furthermore, according to the American Association of Retired Persons, $89 \%$ of the people above 50 want to receive healthcare in their homes due to difficulties in visiting medical centers. In their work [17], the authors propose to use drones in order to overcome such problems in rural areas and for elderly living in remote areas, providing a very interesting AAL solution.

Drones can be used to deliver routine test kits, medication refills and even pick up standard blood and urine tests. Such an application will immensely reduce travel time and workloads of caregiver and medical personnel. Drones are more competitive when time-sensitive tasks and goods are considered and are also independent of the ground roads and terrain. However, while such a solution will definitely contribute to extending the AAL systems in providing sustainable care for the elderly in their selected living environments, it is not as simple as it looks. The authors propose and detail the concept of drone-aided aerial healthcare delivery and pickup service for chronic disease and elderly people in remote/rural areas. The main architecture of the system is given in Fig. 4 below.

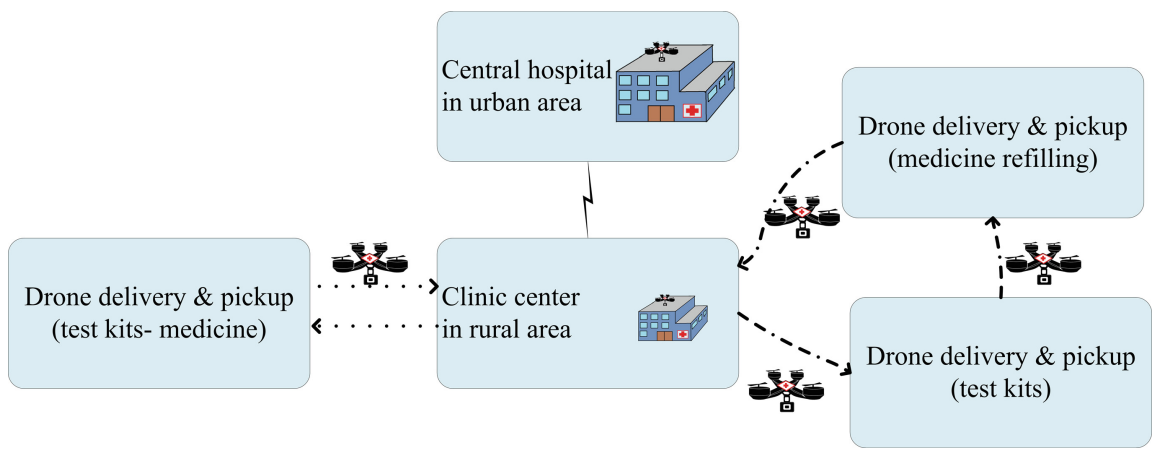

Fig. 4. Concept of drone-aided healthcare delivery and pickup service in rural area

The authors of [17] define and investigate two planning models that can allow the concept to be practically realized: a strategic model and an operational model. The strategic model allows deciding on the optimal location of drone centers and how many centers would be required for a given area, with a specific population and geographical terrain limitations. The algorithm proposed ensures that drones can reach all people regardless of demand levels and number of chronic diseases. The operational model on the other hand determines the optimal number of drones for each center as well as the optimal drone flight schedule for each center. The schedules are defined taking into consideration real life limitations like specific demands of the patients in terms of flying times and restrictions as well as the cost-benefit ratio to help provide an economically viable healthcare solution. The proposed decision making process and the factors taken into consideration by the authors are given in Fig. 5 below. 


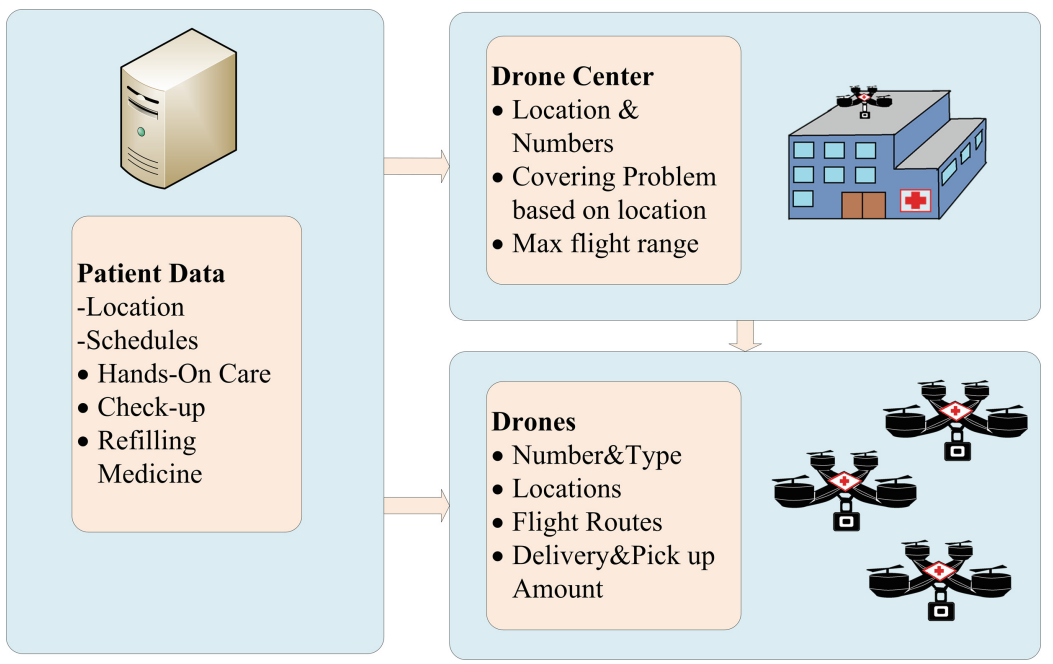

Fig. 5. Decision-making process

Based on collected data, including patient schedules for medical center care, regular check-up schedules, medicine refills, residency information etc. patients can be divided in two main categories: one which needs in-person hands-on care and one which only needs regular testing and medication replenish. The models and the provided solution algorithms address exclusively the second group of people. The authors carry out an illustrational cost-benefit analysis which can be used as a decision process for the stake holders. The suggested approach can be further extended to include variable flying times taking into consideration the battery consumption of the drones.

A major health problem that might lead to death especially in elderly people is cardiac arrest. An interesting niche application addressing distribution and delivery of AEDs (Automated External Defibrillators) which incorporates drones is discussed in the thesis work of Lennartsson [18]. When a cardiac arrest happens outside of a hospital the survival rate is very low, because the person has to be shocked with a defibrillator within minutes of the attack. The survival rate is said to be $74 \%$ if the patient is shocked within $3 \mathrm{~min}$ of the arrest [19] and is reduced to $50 \%$ after $5 \mathrm{~min}$ [20]. As a rule, defibrillators are carried in all ambulances and recently, in Sweden, there are AEDs placed in some public areas. However, as the study in [18] points out the average time for an ambulance to reach the patient in Stockholm is around $13 \mathrm{~min}$. Even worse, there are many islands, where ambulances have difficulty to reach. In such situations, the authors propose the use of drones. Their extensive study investigates the records to determine the spots where cardiac arrest occurrences in Stockholm are difficult to reach by ambulances and evaluate whether drones can be used to arrive before the EMS system. The drone used in the tests is Deficopter, a drone carrying an AED [21] that can travel at a speed of $70 \mathrm{~km} / \mathrm{h}$ within a radius of $10 \mathrm{~km}$. When an emergency call arrives, the Deficopter is programed with the location information and sent out. The on-board camera allows the emergency personnel to spot the scene and 
drop the AED in a suitable location by using a parachute. The study reveals some very interesting results.

First, based on available document records, the authors investigate the density of OHCD (Out of Hospital Cardiac Arrest) cases and determine the 10 best (optimal) places for placing the AEDs in the larger Stockholm area. Then using different GIS data (Geographical Information system) they compare the results for the number of cases the drone would have arrived faster than an ambulance in an 8.5 min radius area, in a 5 min radius and in a 3 min radius area. They consider two different scenarios - the so called 50/50 in which the two parameters - high density of OHCD cases and ambulance arrival time interval - are weighed equally; the 80/20 scenario in which the weights of the above parameters were respectively $80 \%$ and $20 \%$. It is interesting to note that while for the $8.5 \mathrm{~min}$ radius case the ambulances were faster, for the critical 3 min radius case the drone definitely performed much better. The two sets of graphs given below relate to the inner Stockholm area (Fig. 6) and the larger Stockholm area (Fig. 7) (including a number of small disconnected islands).

The second important observation made is that when the larger, less connected area is considered the drones definitely outperform the ambulances for the $5 \mathrm{~min}$ and the 3 min intervals. Despite the possibility of errors arising from the ArcGIS tools and the tuning of the parameters mentioned above these data show that drones can fit quite well in very specific niche AALS applications which other applications cannot cover. The idea of creating a network of AED centers, to cover different neighborhoods in and around large cities and maybe remote rural areas is yet another step towards making the immediate home and residential neighborhoods more convenient and safe for the elderly, thus in a sense extending the AAL concept.

Similar use of drones for delivery of defibrillators in the Netherlands is described in [22].

A step further in this line of work is the research presented in [23] which reviews the current status of innovative drone delivery with a particular emphasis on healthcare. It proposes two new models associated with the design of a drone healthcare delivery network which will facilitate timely, efficient and more economical drone healthcare delivery to potentially save lives and in the long run extend supportive living environments even further. The authors have compiled a comparative summary with data about the payload the drone can carry, its flying range and speed which is given below in Table 4.

In their work they consider the following scenario: emergency medical supplies need to be delivered to an outlying area that is not completely served by good roads but is too far for drone delivery alone. Such a case requires a tandem solution. For the first leg of the solution land based transportation is used to deliver the supplies/medications from a warehouse to the so called "drone nest", which is in sufficient proximity to the area/person in need. For the second leg usage of drones is suggested from the drone nest to the required precise location/person. For this general scenario two models are proposed and investigated: the first one has the objective to minimize the total weighted delivery time per drone nest; the second model aims to minimize the weighted time to 
The number of cases the drone would have covered within a 8.5 minute radius

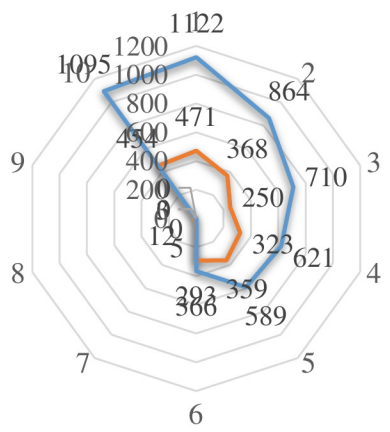

The number of cases the drone would have covered within a 5 minute radius

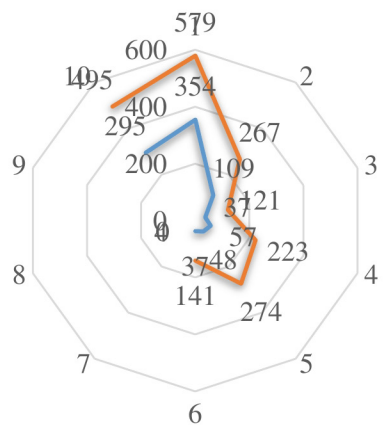

The number of cases the drone would have covered within a 3 minute radius

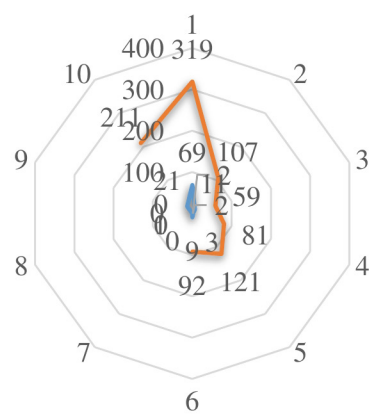

- Number of cases where the drone would have been faster

- Number of cases where the drone would not have been faster

Fig. 6. The number of cases covered by drone/ambulance within $8.5,5$ and 3 min radius for inner Stockholm area 
The number of cases the drone would have covered within a 8.5 minute radius

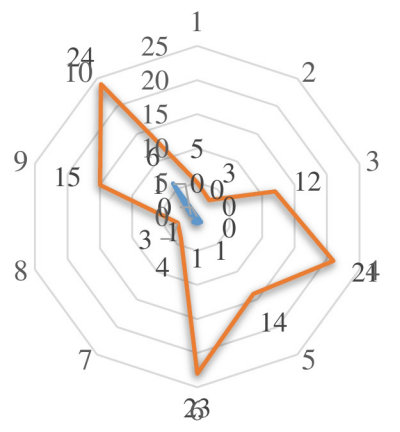

The number of cases the drone would have covered within a 5 minute radius

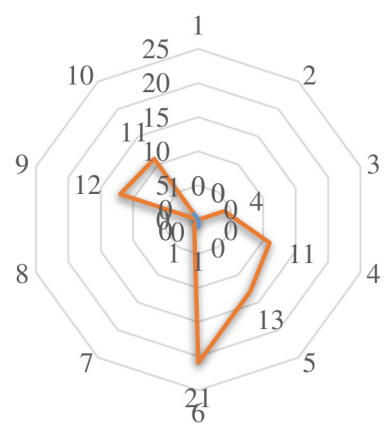

The number of cases the drone would have covered within a 3 minute radius

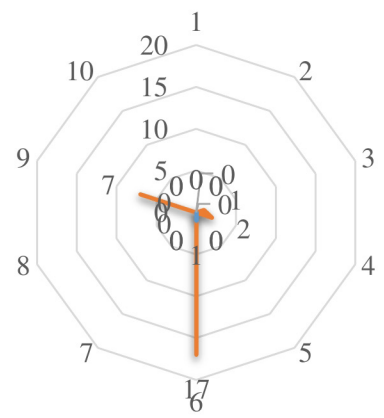

- Number of cases where the drone would have been faster

Number of cases where the drone would not have been faster

Fig. 7. The number of cases covered by drone/ambulance within $8.5,5$ and 3 min radius for larger Stockholm area 
Table 4. Summary of drone specifications

\begin{tabular}{l|l|l|l}
\hline Drone model/company & Payload & Flying range & Max speed \\
\hline Matternet & $2 \mathrm{~kg}$ & $10 \mathrm{~km}$ & $40 \mathrm{~km} / \mathrm{h}$ \\
\hline DHL parcel & $2 \mathrm{~kg}$ & $12 \mathrm{~km}$ & $>40 \mathrm{~km} / \mathrm{h}$ \\
\hline Zipline & $1.35 \mathrm{~kg}$ & $72 \mathrm{~km}$ & $144 \mathrm{~km} / \mathrm{h}$ \\
\hline Flirtey & $2 \mathrm{~kg}$ & $32 \mathrm{~km}$ & - \\
\hline Delft University & $4 \mathrm{~kg}$ & $12 \mathrm{~km}$ & $96 \mathrm{~km} / \mathrm{h}$ \\
\hline
\end{tabular}

deliver to all demand points in a given area. Optimization functions are determined for the two models and the results of the simulations are given in terms of the "investment over delivery distance" tradeoff or similarly "investment over delivery time" tradeoff. The models use a budget constraint while providing location decisions for warehouses and drone nests that enable timely delivery. Since time is of essential importance in an emergency, faster response would prevent medical trauma and potentially save lives. The authors believe that such models will be especially useful not only in developed countries where road congestion or bad weather conditions can prevent servicing the "last mile" to the patient but also in developing countries i.e. African countries, where a large percentage of the population is not served by all year round operable roads.

\subsection{Drone Aided Safety Related Applications}

A common safety hazard for the elderly, especially people in early stages of dementia, is getting lost or wandering off. Some very interesting IoT and drone based solutions in this respect in recent years can be found in [24, 25].

The first work presents a detailed review of the subject of hazardous wandering and the existing technological solutions on wandering in the context of AAL for the elderly. An elaborate definition of wandering and a classification of wandering models are given followed by the examination of existing solutions for managing wandering. Different approaches for managing wandering range from event-monitoring-based wandering discovery, trajectory-tracking-based wandering detection to location combined with Geofence-based prevention of wandering-related adverse results. Building up on the definition, categorization, and the state of the art, the authors discuss major research challenges and future directions in detecting wandering locomotion in different settings. A detailed definition of wandering is cited from [26], Mapping the maze of terms and definitions in dementia-related wandering.

In this consequential work, the definition of wandering is given as follows: "A syndrome of dementia-related locomotion behavior having a frequent, repetitive, temporally disordered, and/or spatially disoriented nature that is manifested in lapping, random, and/or pacing patterns, some of which are associated with eloping, eloping attempts, or getting lost unless accompanied". 
Wandering is so very difficult to define because it can take so many different forms: sometimes it is expressed in pacing back and forth between two points which do not have to be necessarily very close to each other; sometimes it is in the form of "lapping", as moving in a circle, visiting points in a sequential or random manner without repetitions. Such behavior, which in general is not necessarily dangerous, can become problematic in the case of the elderly; it may even lead to injuries and quite serious negative consequences. So far research in this area has focused mainly on wandering evaluation and wandering detection. In principle, the evaluation process involves recognition and testing of wandering movements. The main objective is to determine patterns and characteristics related to wandering based on an offline analysis of trajectory data collected from sensors, in most cases predominantly indoors. Wandering detection on the other hand aims for the development of assistive systems to provide safety assurance for patients with dementia considering both indoor and outdoor environments. In [24], the authors group the existing solutions in 3 major categories. The first one relates to systems that provide event based wandering discovery [27-31]. The second is called "trajectory-tracking-based wandering discovery" [32-39]; while the third category summarizes works on location-based prevention of wandering related adverse events [40-62]. A major research challenge is posed by the fact that wandering can appear in a very great variety of forms and the data collected so far is insufficient for comprehensive modelling. Studying the correlation between wandering and its related factors is a very crucial for in-depth analysis and mandates the use of elaborate statistical data mining methods. However, the authors believe that a possible solution lies not in designing isolated assistive systems but in enhancing human computer interaction in AALS for training and guiding such patients in order to reduce possible health risks and hazardous situations.

An interesting drone based application has been proposed that can help in cases of wandering dementia patients [25]. A team from Toronto University describes 3 different experiments involving the use of UAV for the purpose of locating a wandering person with dementia. The exact experiments are performed on test subjects simulating individual lost patients with dementia (PwD) employing drones together with Search and Rescue (SAR) operational methods for their rescue. Specific algorithms to determine the drones' paths are proposed and tested. Performance metrics include the time needed to detect the lost person and the complete duration of each mission. Furthermore the authors provide a differential longitude and latitude analysis from an initial parting point (IPP) and for that calculate the time to find the test subject and the battery life of the drone.

The first experiment is carried out in the area around the City of Hamilton, Ontario. Three attempts are made with different pilots and different drones (DGI Mavic Drone, Aeryon Labs Ranger Drone and a drone designed by IMR systems). The first attempt with DGI Mavic (743 g, 36 km/h, $5000 \mathrm{~m}$ above sea level, Field of View 78 $)$ and the second attempt (Aeryon Labs Ranger Drone) use camera feed displayed on a mobile phone and are unsuccessful due to the poor picture quality on the mobile device. The third attempt (IMR Systems Drone) finds the person in 6:02 min. The second 
experiment is conducted in an open urban park with a drone DJI Phantom 4 Pro (1388 g, $72 \mathrm{~km} / \mathrm{h}, 6000 \mathrm{~m}$ above sea level, Field of View $\left.84^{\circ}\right)$. The problem with this experiment is that regulations in Canada pose limitations on the areas that drones can be flown. Parks and recreation areas are restricted areas. So the experiment is carried out in a similar close location and the test subject is found in less than $10 \mathrm{~min}$. The final experiment is carried out in the same area as the second one and uses the same drone DJI Phantom 4 Pro this time controlled through the DJI Go 4 app (v4.1.5) on an iPhone $5 \mathrm{~s}$ to make use of the software's features and to record the flight data. Its main focus is determining the differential latitude from the IPP and its relation to the time for discovering the target.

These experiments bring light to several very important issues related to the use of drone for tracking and locating wandering elderly people in outdoor areas. First of all, the authors are optimistic that the suggested approach allows locating the missing person within the first 10 min which is considered as the lower limit for safety independent of the terrain. Second, the authors point out the many major hurdles that have to be overcome before such operations can be considered of practical value. The first one is that in order to use drones for these purposes models based on underlying algorithms of wandering that can be technically implemented to automate the search process are required. Another important issue is that drones can help locate the person but monitoring battery usage as well as longitude and latitude is crucial for success. And last but not least, a major problem exists with the specific regulations and restrictions for flying drones in different countries which have to be clarified before drone-based search and localization can become a practically feasible option.

\subsection{Drone Aided Daily Activities and Routines}

Even though drones are more often associated with outdoor activities there is some interesting research on using UAV in indoor locations.

The first application is a UAV for indoor patient care, called Healthbuddy, which proposes a customized quadcopter design to provide assistive healthcare at home [63]. Its main goal is to facilitate independent living for the elderly in their living quarters. The application can help people suffering from long hours of isolation, both elderly, or cancer patients or patients with depression. Healthbuddy is designed to navigate around the house at regular intervals trying to detect the person/patient using image, sonar and voice recognition (VR) strategies. The drone can avoid collisions with walls and different objects in real-time. Once the drone locates and recognizes the person/patient it starts a wireless communication with a server and receives further instructions based on queries. Health based queries are designed to require simple yes/no responses; VR strategies are used to classify the speech responses extracted from surrounding noise; using the collected information the server provides patient analysis; and finally the Healthbuddy system as a whole determines what is the most appropriate line of action in the given situation. 
The functional block diagram of Healthbuddy is given in Fig. 8 below.

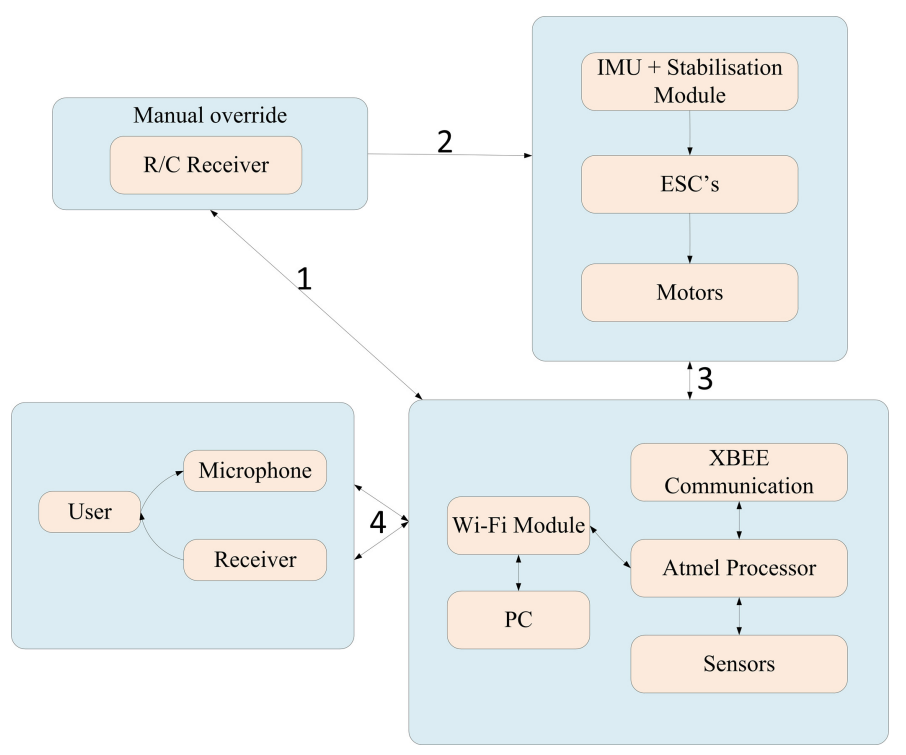

Fig. 8. The block diagram of Healthbuddy

The main hardware components comprise the AeroQuad Kit (Arduino Mega 2560) gyroscope, 3-axis accelerometer, magnetometer, barometer sensor and an ultrasonic sensor. Communication components include a VR Shield (EasyVR Shield 2.0), a speaker and an amplifier, power source (Lipo Battery Pack $5000 \mathrm{mAh}$ ); the wireless communication network components are ZigBee modules (the XBee Pro $60 \mathrm{~mW}$ ), the XBee Explorer Dongle, and the ITEAD XBee Shield. Since the work is still in its development stage there aren't final published results but efforts are being put in combining different algorithmic solutions from literature. The authors believe that with time, the promising solutions existing separately in literature could be combined to form a unique platform to serve the objectives of their drone based AAL project.

A more evolved work on indoor drone application is presented in "QuadAALper The Ambient Assisted Living Quadcopter", as the PhD thesis of Ricardo Miguel Gradim Nascimento from the University of Porto [64].

The work presents the design of a drone that can autonomously navigate inside the house and recognize a person lying on the floor. The authors propose an original method for flying the drone indoors without GPS information. Their method is based on QR code detection using a smartphone mounted on top of the drone. The QR codes, $20 \times 20$ are placed on the ceiling. The drone is a Arducopter, controlled by an open source controller Pixhawk. The mobile phone used in the project is a HTC One M8, equipped with a $2.5 \mathrm{GHz}$ processor and a Duo Rear Camera, with a $2600 \mathrm{mAh}$ battery. 
The developers make full use of all the sensors coming with the mobile phone (gyroscope, accelerometer, proximity, compass and barometer) and add external sonar sensor and four Infra-Red sensors. The main algorithms use the OpenCV and Zxing libraries. From communication point of view, the two major protocols used are the NMEA and the MAVLink protocol. The NMEA protocol [65] is a combined electrical and data specification for communication between electronic devices like sonars, autopilot, GPS receivers and other types of instruments. In general, programs providing real time localization work with data in the NMEA format. The Pixhawk, the drone control unit used in the project also accepts NMEA data. The MAVLink (MAVLink Micro Air Vehicle Communication Protocol) [66] protocol enables communication between the mounted Android smartphone and the drone control module (Pixhawk) for mission planning, for receiving live telemetric data and for monitoring the Pixhawk status.

Tests have been carried out which prove that the suggested method is quite accurate and allows the drone to correctly capture and decode the QR code at angles of $45 \%$ and $30 \%$, in different light environments (bright, medium, dark) and for different mobility of the drone. Main advantage of the proposed method is its simplicity as compared to other SLAM (Simultaneous Localization and Mapping) algorithms. Furthermore the authors add person recognition algorithms and are successful in detecting and recognizing a person lying on the floor. Even though this work is very elaborate, detailed and much more advanced in terms of practical realization than other similar attempts, there is still a lot to be done before a commercial drone can become the Flying Home Buddy of the elderly.

\subsection{Drones for Better Social Inclusion}

Many people can argue that computer games and virtually reality are one of major reason for social isolation among the younger generation. Obviously there is also the other camp which points out the positive effects of this modern pastime as well as its potential for enhancing both visual and motor skills. One group of researchers from Singapore has put these opinions to a test, not with children or adolescents, but with elderly citizens in a nursing facility, with ages ranging from 56 to 92 years [67].

They carried out an intervention project using Nintendo Wii to explore the longterm physiological effects of video games on the elderly. The intervention was a 6 weeks long program, carried out with two groups of people: one playing computer games the other playing conventional games for the same period every day. They measured loneliness (using UCLA Loneliness Scale), self-esteem (using Rosenberg Self-esteem Scale), affect (using Brandburn Affect Balance Scale). Amazingly, on all 3 metrics the Nintendo Wii group scored better; significantly higher on self-esteem and affect and significantly lower on loneliness as compared to the control group. Other literature on this subject can be found in [68-70].

This and some other similar studies inspired a group from the Polytechnic Institute of Leiria, Portugal together with a group from Ecuador, (Universidad de las Fuerzas Armadas Espe) to develop a drone based VR application for the elderly [71, 72]. 
Their work makes use of different wireless technologies, wearable and inertia measurement unit (IMU) sensors to generate a body area network that scans arm movement and sends information through Bluetooth Low Energy (BLE) to control a virtual UAV (Unmanned Aerial Vehicle) remotely. The developed system consists of a 3D simulator using VR glasses for immersive visualization and Raspberry Pi devices with Sense HAT board for hand controls. As a result, it emulates the control of a drone in a simulated environment. The general architecture of the system is given in the Fig. 9 below.

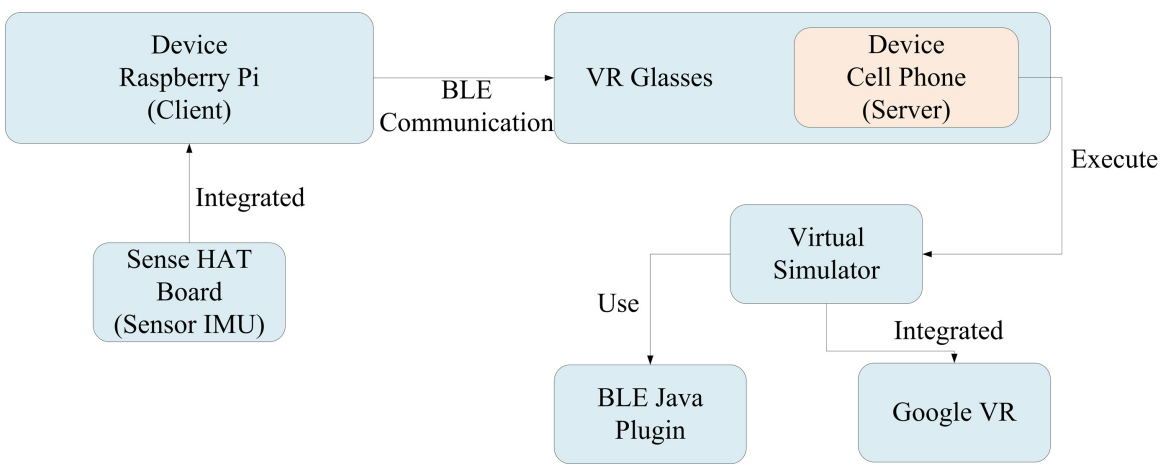

Fig. 9. General architecture of drone-based VR application

For practically implementing this system with elderly residents the researchers developed 2 visualization modes and 2 difficulty modes. The visualization modes determine the perspective in which the user looks at the environments. One possible perspective is to look as a third person - i.e. the user can both see and control the drone. The second perspective is that of first person - i.e. the user sees the environment through the eyes of the drone. Two separate difficulty levels are designed - easy, where only 4 movements are allowed and difficult, where the user has to make 6 different movements with both hands to control the drone. The application helps the elderly preserve and/or develop better cognitive skills (eye-hand coordination) and improve mental and physical state. The researchers performed tests with a group of elderly in a nursing facility and observed that their skill level increased when they used the system for a period of time, progressing from easy to difficult level. The proposed system is still a first prototype and further improvements can be made to increase the involvement of the players. More and longer tests are also required but the first results are really optimistic. Well it might not be surprising that there are quite some things in common between the children and the elderly. As Shakespeare put is centuries ago in his speech "Al the world's a stage" - "...one man in his time plays many parts, ...Last scene of all, that ends this strange eventful history, is second childishness and mere oblivion." 


\subsection{Challenges and Discussion}

The development of new technologies, new materials and technical solutions allows increasing the quality and versatility of the AALs for the elderly. Each new technology brings new possibilities for more elaborate and unobtrusive ways to protect the elderly and allow them to continue their fulfilling, dignified and independent living. In this chapter we introduced a new technology, the Unmanned Aerial Vehicles (UAV), or simply "the drones" and discussed its possible application in AALS. In the last decade UAVs have drawn a lot of interest from the academic and industrial sectors specifically because of their possibilities in the area of military applications. In the last several years however, a number of niche applications have been developed which open the way for using UAVs in different AALS. Thus the chapter focused on classifying and describing these very recent developments.

Different aspects of AALS like the health monitoring, the safety, the daily routines support and the social connectedness aspect were examined and applications and projects from literature were summarized. All of discussed applications are in their initial development stage, they are quite innovative, address very specific and intriguing cases and evaluating them is not a simple task. What can be done at this early stage is to point out the main challenges they present in terms of technical requirements and suggest some plausible evaluation criteria for the exploitation phase.

Considering the technical requirements UAV based systems for AAL applications have several very important features in common. They should definitely be designed for failure, should possess high degree of dynamism and adaptability to different specific conditions, should allow for ease of deployment and most of all should be able to ensure a desired level of privacy and confidentiality. Last but not least they should provide simple and easy-to-understand user interfaces since most of the elderly have lower levels of technical skills and acceptance of new technologies.

The most important evaluation angle of such systems is how well they are accepted by the targeted population group. All of the drone based projects discussed in this chapter address a specific niche application and provide an interesting non-traditional solution. Since most of the projects are either in their initial state or first prototype it is very difficult to evaluate the effect they will have on the elderly. That is why, instead of directly evaluating them we would like to consider some important criteria that the evaluation process should include. Naturally these criteria are user oriented i.e. subjective and their determination requires a minimum period over which the given system has been tested in practice. As mentioned before the projects discussed in this chapter are in their very initial stage so the evaluation criteria summarized below should be understood more in the sense of setting a roadmap for researchers working in this field than as a direct evaluation of the presented applications.

One of the most comprehensive assessments related to AALS is suggested in [73]. Based on that, the major main evaluation parameters are summarized below (Table 5). 
Table 5. Main evaluation parameters

\begin{tabular}{l|l}
\hline $\begin{array}{l}\text { Evaluation } \\
\text { criteria }\end{array}$ & Definition \\
\hline Usability & $\begin{array}{l}\text { The extent to which a technological product can be used by the specified } \\
\text { user group to achieve the specified goal in an effective way }\end{array}$ \\
\hline Acceptability & $\begin{array}{l}\text { The degree of primary users predisposition to carry out activities using the } \\
\text { intended system/device }\end{array}$ \\
\hline Efficacy & $\begin{array}{l}\text { The capability of the users to effectively complete tasks and achieve goal } \\
\text { using the specific tool or system }\end{array}$ \\
\hline Utility & $\begin{array}{l}\text { The degree to which users believe that using a particular system would } \\
\text { enhance their performance }\end{array}$ \\
\hline Obtrusiveness & $\begin{array}{l}\text { The degree of device caused encumbrance as perceived by users on } \\
\text { themselves or in their environment }\end{array}$ \\
\hline
\end{tabular}

These criteria provide an exhaustive evaluation of a given system/technology and will be very useful if applied to todays and future AAL systems. However, the task is not as simple as it looks. Only the first criteria (usability) can partially be evaluated in an objective way, while all the others are related to the subjective experience of the user. This means that a proposed solution has to be put into practice and endure a specific evaluation period before reliable results can be obtained.

Another evaluation approach can be created considering the ideas proposed in [11]. The authors' baseline is that user's acceptance of personal space modifications depends on the user's needs and lifestyle preferences. They classified the developments in AALS into three groups: ambient-intelligent space (AmI-S), physical space (PS), and virtual space (VS) - integrated together to support independent life. Currently, there is a lot of interest for more detailed investigations on the linkage between AALS and user's lifestyles. Thus the discussed drone based systems can be evaluated in terms of their contribution to this so called "lifestyle change model".

At the current state of life we can only evaluate the proposed systems as singular, niche applications which if proven fiscally viable can pave the road to enhancing the AALS even more. Drones can fill in gaps where other technologies cannot (delivery functions), can extend scope of existing applications (localization and rescue of wandering $\mathrm{PwD}$ ), or can enrich in content existing systems (virtual reality and gaming) and make them more appealing and easily acceptable for the elderly.

However, on the flip side, the drone technology is still not mature and feasible enough to be easily deployed on an everyday scale. There are a number of challenges and hurdles to be overcome. Roughly they can be divided into two groups: regulational and ethic challenges.

Many of the described applications are based on technologically mature solutions and promise to draw even more interest in the future. However, there are several major regulation challenges related to them:

- There is a definite lack of clear nationwide and international regulations related to how, when and where drones be flown and this is quite an important challenge. At the same time more research is required looking into existing (even though scatted and quite limited) regulations and how they have to be altered. 
- Regulations in many countries do not allow for a drone above certain size and weight to be flown just by anybody; solutions require the creation and support of major organizational structures behind. It also mandates defining specific protocols regarding the planning of the flight and areas restricted for flying.

- As drones become more sophisticated and powerful questions of their proper management become even more important not only within the single countries but also internationally. Thus regulations have to be created and implemented at a much larger scale.

The second major aspect is the ethical aspect. The concepts of personal space and immediate environment are gaining completely new meanings and it has becomes even more difficult and elusive to define the borders of what is "good" and what is "bad". Since this chapter is focused more on the technological aspect details on the ethical aspect are given. A very good material on this subject can be found in [74].

\section{Conclusion}

Unmanned Aerial Vehicles (UAVs) simply known as "drones" have been a hot research issue for the last decade, mainly due to their attractive usage possibilities in the military and network communication areas. However, very recently, several groups of researchers from all different parts of the world have turned their attention to a new application area for this emerging technology - the ambient assisted living systems especially targeting the elderly. Quickly increasing percentage of the aging population is placing higher and more extensive demands on our society; meeting the goals with traditional methods places an extensive burden both in terms of human resources and financial structures. Thus, there is a large stream of scientific research focused on how to use emerging technologies to ease this burden and make life better, easier, more secure and fulfilling for the elderly. Many solutions have been offered so far but still there are niche applications which can have better solutions. Some of these very recent applications, which offer innovative AALS solutions based on the use of drones have been summarized and discussed in this chapter. Without trying to be exhaustive, the chapter covers major issues on the structure and principle of operation of drones, and provides information on recently published results from projects that utilize drones to improve the life, security, environment and connectedness of the elderly. Finally the authors discuss some major challenges and provide guidelines for user perspective evaluation of such systems in the future.

\section{References}

1. Glass Company Homepage. https://www.x.company/glass. Accessed 31 Aug 2018

2. Blackman, S., et al.: Ambient assisted living technologies for aging well: a scoping review. J. Intell. Syst. 25(1), 55-64 (2015)

3. Wan, J., Gu, X., Chen, L., Wang, J.: Internet of Things for ambient assisted living: challenges and future opportunities. In: 2017 International Conference on Cyber-Enabled Distributed Computing and Knowledge Discovery, Nanjing, pp. 354-357 (2017) 
4. Rashidi, P., Mihailidis, A.: A survey on ambient-assisted living tools for older adults. IEEE J. Biomed. Health Inform. 17(3), 579-590 (2013)

5. McCombie, D.B., Shaltis, P.A., Reisner, A.T., Asada, H.H.: Adaptive hydrostatic blood pressure calibration: development of wearable, autonomous pulse wave velocity blood pressure monitor. In: Conference Proceedings of IEEE Engineering in Medicine and Biology Society, pp. 370-373 (2007)

6. Hasdemir, İ., Ertaş, G.: Experimental analysis of optical sensors in detecting heart beat. In: 2017 Medical Technologies National Congress (TIPTEKNO), Trabzon, pp. 1-4 (2017)

7. Demir, E., Köseoğlu, E., Sokullu, R., Şeker, B.: Smart home assistant for ambient assisted living of elderly people with dementia. In: International Workshop on IoT, M2M and Healthcare, Lund, pp. 609-614 (2017)

8. Lloret, J., Canovas, A., Sendra, S., Parra, L.: A smart communication architecture for ambient assisted living. IEEE Commun. Mag. 53(1), 26-33 (2015)

9. Skocir, P., Krivic, P., Tomeljak, M., Kusek, M., Jezic, G.: Activity detection in smart home environment. In: 20th International Conference on Knowledge and Intelligent Information and Engineering System, pp. 672-681 (2016)

10. Fuxreiter, T., Mayer, C., Hanke, S., Gira, M., Sili, M., Kropf, J.: A modular platform for event recognition in smart homes. In: 12th IEEE International Conference on e-Health Networking, Applications and Services, pp. 1-6 (2010)

11. Al-Shaqi, R., Mourshed, M., Rezgui, Y.: Progress in ambient assisted systems for independent living by the elderly. Springerplus 5(624), 1-20 (2016)

12. Rakhman, A.Z., Kurnianingsih, Nugroho, L.E., Widyawan: u-FASt: ubiquitous fall detection and alert system for elderly people in smart home environment. In: Makassar International Conference on Electrical Engineering and Informatics, pp. 136-140 (2014)

13. Kong, X., Meng, L., Tomiyama, H.: Fall detection for elderly persons using a depth camera. In: International Conference on Advanced Mechatronic Systems, pp. 269-273 (2017)

14. Bhati, N.: mHealth based ubiquitous fall detection for elderly people. In: 8th International Conference on Computing, Communication and Networking Technologies, pp. 1-7 (2017)

15. Epping-Jordan, J., Pruitt, S., Bengoa, R., Wagner, E.: Improving the quality of health care for chronic conditions. Qual. Saf. Health Care 13(4), 299-305 (2004). Ward, B.W.: Multiple chronic conditions among us adults: A 2012 update (2014)

16. Gerteis, J., et al.: Multiple chronic conditions chartbook. Agency for Healthcare Research and Quality (2014)

17. Kim, S.J., Lim, G.J., Cho, J., Cote, M.J.: Drone-aided healthcare services for patients with chronic diseases in rural areas. J. Intell. Robot. Syst. 88, 163-180 (2017)

18. Lennartsson, J.: Strategic placement of ambulance drones for delivering defibrillators to out of hospital cardiac arrest victims. Stockholm, KTH Royal Institute of Technology (2015)

19. Valenzuela, T.D., Roe, D.J., Nichol, G., Clark, L.L., Spaite, D.W., Hardman, R.G.: Outcomes of rapid defibrillation by security officers after cardiac arrest in casinos. New Engl. J. Med. 343(17), 1206-1209 (2000)

20. Hjärtstartarregistret. https://www.hjartstartarregistret.se/\#/faktasida/1. Accessed 31 Aug 2018

21. The Verge. Health from above: a drone to deliver defibrillators to heart attack victims. http:// www.theverge.com/2013/8/24/4654514/definetz-height-tech-defibrillator-carrying-drone-ingermany. Accessed 31 Aug 2018

22. Prigg, M.: The ambulance drone that could save your life. http://www.dailymail.co.uk/sc iencetech/article-2811851/The-ambulance-drone-save-life-Flying-defibrillator-reach-speeds60mph.html. Accessed 31 Aug 2018

23. Scott, J.E., Scott, C.H.: Drone delivery models for healthcare. In: Proceedings of the 50th Hawaii International Conference on System Sciences, pp. 3297-3304 (2017) 
24. Lin, Q., Zhang, D., Chen, L., Ni, H., Zhou, X.: Managing elders' wandering behaviour using sensors-based solutions: a survey. Int. J. Gerontol. 8, 49-55 (2014)

25. Hanna, D., Ferworn, A., Lukaczyn, M., Abhari, A., Lum, J.: Using UAVs in locating wandering patients with dementia. In: IEEE/ION Position, Location and Navigation Symposium (2018)

26. Algase, D.L., Moore, D.H., Vandeweerd, C.: Mapping the maze of terms and definitions in dementia-related wandering. Aging Mental Health 11(6), 686-689 (2007)

27. Doughtyt, K., Williams, G., King, P.J., et al.: DIANA - a telecare system for supporting dementia sufferers in the community. In: Proceedings EMBC, pp. 1980-1983 (1998)

28. Ota, K., Ota, Y., Otsu, M., et al.: Elderly-care motion sensor using UWB-IR. In: IEEE Sensors Applications Symposium (SAS 2011), pp. 159-162 (2011)

29. Masuda, Y., Yoshimura, T., Nakajima, K., et al.: Unconstrained monitoring of prevention of wandering the elderly. In: Proceedings of the 24th Annual Conference and the Annual Fall Meeting of the Biomedical Engineering Society EMBS/BMES Conference (EMBS/BMES 2002), pp. 1906-1907 (2002)

30. Jit, B., Zhang, D.Q., Qiao, G.P., et al.: A system for activity monitoring and patient tracking in a smart hospital. In: Proceedings of the 4th International Conference on Smart Homes and Health Telematics (ICOST 2006), pp. 196-203 (2006)

31. Rowe, M., Lane, S., Phipps, C.: CareWatch: a home monitoring system for use in homes of persons with cognitive impairment. Top Geriatr. Rehabil. 23, 3-8 (2007)

32. Martino-Saltzman, D., Blasch, B.B., Morris, R.D., et al.: Travel behavior of nursing home residents perceived as wanderers and nonwanderers. Gerontologist 31, 666-672 (1991)

33. Algase, D.L., Beattie, E.R., Leitsch, S.A., et al.: Biomechanical activity devices to index wandering behaviour in dementia. Am. J. Alzheimers Dis. Other Demen. 18, 85-92 (2003)

34. Kearns, W.D., Nams, V., Fozard, J.: Tortuosity in movement paths is related to cognitive impairment. Wireless fractal estimation in assisted living facility residents. Methods Inf. Med. 49, 592-598 (2010)

35. Kearns, W.D., Algase, D., Moore, D.H., et al.: Ultra wideband radio: a novel method for measuring wandering in persons with dementia. Gerontechnology 7, 48-57 (2008)

36. Kearns, W.D., Fozard, J.L.: Evaluation of wandering by residents in an assisted living facility (ALF) using ultra-wide band radio RTLS. J. Nutr. Health Aging 13, S54 (2009)

37. Kearns, W.D., Fozard, J.L., Nams, V.O., et al.: Wireless telesurveillance system for detecting dementia. Gerontechnology 10, 90-102 (2011)

38. Kearns, W.D., Fozard, J.L., Becker, M., et al.: Path tortuosity in everyday movements of elderly persons increases fall prediction beyond knowledge of fall history, medication use, and standardized gait and balance assessments. J. Am. Med. Dir. Assoc. 13(7), 665.e7-665. e13 (2012)

39. Nams, V.O., Bourgeois, M.: Fractal analysis measures habitat use at different spatial scales: an example with American marten. Can. J. Zool. 82, 1738-1747 (2004)

40. Vuong, N.K., Chan, S., Lau, C.T., et al.: A predictive location-aware algorithm for dementia care. In: Proceedings of the 15th IEEE International Symposium on Consumer Electronics (ISCE 2011), pp. 339-342 (2011)

41. Sposaro, F., Danielson, J., Tyson, G.: iWander: an android application for dementia patients. In: Proceedings of the 32nd Annual International Conference of the IEEE Engineering in Medicine and Biology Society (EMBS 2010), pp. 3875-3878 (2010)

42. Wan, J., Byrne, C., O'Hare, G.M.P., et al.: Orange alerts: lessons from an outdoor case study. In: Proceedings of the 5th International ICST Conference on Pervasive Computing Technologies for Healthcare (Pervasive Health 2011), pp. 446-451 (2011) 
43. Wan, J., Byrne, C., O'Hare, G.M.P., O’Grady, M.J.: OutCare: supporting dementia patients in outdoor scenarios. In: Setchi, R., Jordanov, I., Howlett, R.J., Jain, L.C. (eds.) KES 2010. LNCS (LNAI), vol. 6279, pp. 365-374. Springer, Heidelberg (2010). https://doi.org/10. 1007/978-3-642-15384-6_39

44. Hoey, J., Yang, X., Quintana, E., et al.: LaCasa: location and context-aware safety assistant. In: Proceedings of the 6th International ICST Conference on Pervasive Computing Technologies for Healthcare (Pervasive Health 2012), pp. 171-174 (2012)

45. Hoey, J., Yang, X., Favela, J.: Decision theoretic, context aware safety assistance for persons who wander. In: Proceedings of the 7th International Workshop on Ubiquitous Health and Wellness (2012)

46. Rodriguez, M., Navarro, R., Favela, J., et al.: An ontological representation model to tailor ambient assisted interventions for wandering. In: AAAI Fall Symposium: Artificial Intelligence for Gerontechnology, AAAI Technical Report, vol. FS-12-01, pp. 32-37. AAAI (2012)

47. Ogawa, H., Yonezawa, Y., Maki, H., et al.: A mobile phone-based safety support system for wandering elderly persons. In: Proceedings of the 26th Annual International Conference of the IEEE Engineering in Medicine and Biology Society, pp. 3316-3317 (2004)

48. Matsuoka, S., Ogawa, H., Maki, H., et al.: 2011. A new safety support system for wandering elderly persons. In: Proceedings of the 33th Annual International Conference of the IEEE Engineering in Medicine and Biology Society, pp. 5232-5235 (2011)

49. Miskelly, F.: Electronic tracking of patients with dementia and wandering using mobile phone technology. Age Ageing 34, 497-499 (2015)

50. Shimizu, K., Kawamura, K., Yamamoto, K.: Location system for dementia wandering. In: Proceedings of the 22nd Annual International Conference of the IEEE Engineering in Medicine and Biology Society (EMBS 2000), pp. 1556-1559 (2000)

51. Lin, C.C., Chiu, M.J., Hsiao, C.C., et al.: Wireless health care service system for elderly with dementia. IEEE Trans. Inf Technol. Biomed. 10, 696-704 (2006)

52. Calvo-Palomino, R., de las Heras-Quirós, P., Santos-Cadenas, J.A., Román-López, R., Izquierdo-Cortázar, D.: Outdoors monitoring of elderly people assisted by compass, GPS and mobile social network. In: Omatu, S., et al. (eds.) IWANN 2009. LNCS, vol. 5518, pp. 808-811. Springer, Heidelberg (2009). https://doi.org/10.1007/978-3-642-02481-8_122

53. Mulvenna, M., Sävenstedt, S., Meiland, F., et al.: Designing and evaluating a cognitive prosthetic for people with mild dementia. In: Proceedings of the 2010 IEEE Energy Conversion Congress and Exposition (ECCE 2010), pp. 11-18 (2010)

54. Lin Q., Zhang D.Q., Huang X.D., et al.: Detecting wandering behavior based on GPS traces for elders with dementia. In: Proceedings of the 12th International Conference on Control, Automation, Robotics and Vision (ICARCV 2012), pp. 672-677 (2012)

55. Wherify Wireless. http://www.mightygps.com/wherify.htm. Accessed 31 Aug 2018

56. Blackburn, P.: Freedom to wander. Nurs. Times 84(49), 54-55 (1988)

57. Miskelly, F.: A novel system of electronic tagging in patients with dementia and wandering. Age Ageing 33, 304-306 (2004)

58. Omnilink. http://www.omnilink.com. Accessed 31 Aug 2018

59. Vivago WristCare. https://www.vivago.com/. Accessed 31 Aug 2018

60. Wong, G.: GPS shoe to track Alzheimer's patients. http://edition.cnn.com/2009/HEALTH/ 06/10/gps.shoes. Accessed 31 Aug 2018

61. Lifelinefl400. http://www.tunstall.co.uk/solutions/lifeline-vi. Accessed 31 Aug 2018

62. TRiLOC. http://www.iloctech.com/. Accessed 31 Aug 2018

63. Todd, C., et al.: A proposed UAV for indoor patient care. Technol. Health Care: Off. J. Eur. Soc. Eng. Med. (2015) 
64. Nascimento, R.M.G.: QuadAALper - The Ambient Assisted Living Quadcopter, Porto (2015)

65. NMEA Data. http://www.gpsinformation.org/dale/nmea.htm. Accessed 31 Aug 2018

66. MAVLink Micro Air Vehicle Communication Protocol - QGroundControl GCS. http:// qgroundcontrol.org/mavlink/start. Accessed 31 Aug 2018

67. Jung, Y., Li, W., Gladys, C., Lee, K.M.: Games for a better life: effects of playing Wii games on the well-being of seniors in a long-term care facility. In: Proceedings of the Sixth Australasian Conference on Interactive Entertainment, pp. 1-6 (2009)

68. Ijsselsteijn, W., Nap, H.H., de Kort, Y., Poels, K.: Digital game design for elderly users. In: The Proceeding of Future Play, Toronto, pp. 17-22 (2007)

69. Cota, T.T., Ishitani, L.: Motivation and benefits of digital games for the elderly: a systematic literature review. Revista Brasileira de Computação Aplicada 7(1), 2-16 (2014)

70. De Schutter, B., Brown, J.A.: Digital games as a source of enjoyment in later life. Games Cult. 11(1-2), 28-52 (2016)

71. Crespo, A.B.: Development a virtual reality model simulation in order to control a drone using a wearable device in a 3D environment, Leiria (2017)

72. Crespo, A.B., Idrovo, G.G., Rogrigues, N., Pereira, A.: Development of a virtual reality model simulation to control a drone by using wearable devices in a 3D environment. In: 1 st International Conference on Technology and Innovation in Sports, Health and Wellbeing (2016)

73. Cavallo, F., Aquilano, M., Arvati, M.: An ambient assisted living approach in designing domiciliary services combined with innovative technologies for patients with Alzheimer's disease: a case study. Am. J. Alzheimer's Dis. Other Dementias 30(1), 69-77 (2015)

74. Novitzky, P.: Ethics of ambient assisted living technologies for persons with dementia, Dublin (2016)

Open Access This chapter is licensed under the terms of the Creative Commons Attribution 4.0 International License (http://creativecommons.org/licenses/by/4.0/), which permits use, sharing, adaptation, distribution and reproduction in any medium or format, as long as you give appropriate credit to the original author(s) and the source, provide a link to the Creative Commons licence and indicate if changes were made.

The images or other third party material in this chapter are included in the chapter's Creative Commons licence, unless indicated otherwise in a credit line to the material. If material is not included in the chapter's Creative Commons licence and your intended use is not permitted by statutory regulation or exceeds the permitted use, you will need to obtain permission directly from the copyright holder.

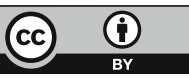

